\title{
An Improved Detection and Classification Technique of Harmonic Signals in Power Distribution by Utilizing Spectrogram
}

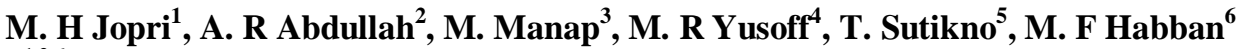 \\ ${ }^{1,2,6}$ Faculty of Electrical Engineering, Universiti Teknikal Malaysia Melaka, Malacca, Malaysia \\ ${ }^{3,4}$ Faculty of Engineering Technology, Universiti Teknikal Malaysia Melaka, Malacca, Malaysia \\ ${ }^{5}$ Department of Electrical Engineering, Universitas Ahmad Dahlan, Yogyakarta, Indonesia
}

\begin{abstract}
Article Info
Article history:

Received Oct 21, 2016

Revised Dec 21, 2016

Accepted Jan 13, 2017

\section{Keyword:}

Classification technique

Detection

Harmonic signal

Time-frequency distributions

Power distribution

Spectrogram

ABSTRACT

This paper introduces an improved detection and classification technique of harmonic signals in power distribution using time-frequency distribution (TFD) analysis which is spectrogram. The spectrogram is an appropriate approach to signify signals in jointly time-frequency domain and known as time frequency representation (TFR). The spectral information of signals can be observed and estimated plainly from TFR due to identify the characteristics of the signals. Based on rule-based classifier and the threshold settings that referred to IEEE Standard 1159 2009, the detection and classification of harmonic signals for 100 unique signals consist of various characteristic of harmonics are carried out successfully. The accuracy of proposed method is examined by using MAPE and the result show that the technique provides high accuracy. In addition, spectrogram also gives 100 percent correct classification of harmonic signals. It is proven that the proposed method is accurate, fast and cost efficient for detecting and classifying harmonic signals in distribution system.
\end{abstract}

Copyright () 2017 Institute of Advanced Engineering and Science. All rights reserved.

\section{Corresponding Author:}

M. H Jopri,

Advanced Digital Signal Processing Group, Faculty of Electrical Engineering, Universiti Teknikal Malaysia Melaka,

Hang Tuah Jaya, 76100 Durian Tunggal, Melaka, Malaysia.

Email: hatta@utem.edu.my

\section{INTRODUCTION}

Power quality (PQ) disturbances analysis specifically for harmonics disturbance turns into an exponentially expanding field of interest. The PQ analysis associates power engineering and power electronics with digital signal-processing, artificial intelligence and optimization techniques [1]. PQ is generally defined as any change in power (voltage, current, or frequency) that interferes with the normal operation of electrical equipment. Furthermore, harmonics and inter-harmonics issues have been identified as one of crucial issue in PQ [2]. To investigate these issues, the information is frequently accessible as a form of sampled time function that is indicated by a time series of amplitudes. At the point when dealing such information, the Fourier transform (FT) based approach is commonly utilized. FT assumes periodicity of a given signal and loses the time axis account; nonetheless, FT not able in providing time information about signal disturbances. Short time Fourier transform (STFT) offers both time and frequency information, nevertheless it suffers severely from the Heisenberg uncertainty principle [2]. The Discrete Fourier transform (DFT) is utilized for analyze frequency content in steady state and it is appropriate for harmonic analysis. Conversely, it is not capable to fast changes in waveform. The DFT has foremost disadvantages such as resolution, spectrum leakage as well as picket-fence effects [1]. The basics of wavelets and wavelet transform can be denoted in [2]. The short time Fourier transforms (STFT) has the restriction of the fixed window 
width, hence it is insufficient for the analysis of the non-stationary harmonic disturbances. The problem of all above signal processing techniques is the principle of Heisenberg's uncertainty in which one cannot distinguish what spectral components be present at what instance of time. The distinctive features that describe harmonic disturbances and methods to extract from logged disturbances are also obtainable [3]. The STFT resolution problems resolved by utilizing wavelet transform (WT. This makes it significantly for tracing changes in signal including fast changes in high-frequency [4]. Awkwardly, WT abilities are often significantly degraded in real practice especially in noisy situations [5]. Based on the discussion, a good method is required due to identify the most perfect, quick and economical way for analysis of harmonic signals.

In this paper, the spectrogram technique which is time frequency analysis technique for distinguishing the signals in time frequency domain is introduced. The spectrogram is a squared magnitude of STFT gives the time waveform energy distribution in joint-time frequency domain where this technique is used in many applications and widely used as an initial investigative tool with less computational complexity, fast and accurate detection and classification technique [6]. The harmonic signals are analyzed and represented in time frequency representation (TFR). Furthermore, the parameters such as RMS and fundamental value, total harmonic distortion (THD), total non-harmonic distortion (TnHD) and total waveform distortion (TWD) for voltage and current are evaluated from TFR and used for the classification of harmonic and inter-harmonic signals. The performance of the proposed technique is verified by classifying 100 signals with numerous characteristics for every type of voltage variation signals and also based on IEEE Std. 1159-2009 [7].

\section{HARMONICS AND INTER-HARMONICS}

According to (IEEE Std. 1159-2009), harmonics which can be caused by rectifiers and inverters, are the inverse of inter-harmonics where they are frequency components of distorted voltages and currents that are integer multiples of the fundamental frequency $(50 \mathrm{~Hz}$ for Malaysia). Harmonic combines with the fundamental voltage or current produces waveform distortion. Harmonic distortion exists due to the nonlinear characteristics of devices and loads on the power system. Meanwhile, Inter-harmonics are frequency components of distorted voltages that are not integer multiples of the fundamental frequency, which resulted from cyclo-converter, arc furnaces, and adjustable speed drives (ASDs) [7]. Inter-harmonics can lead to excitation of low frequency mechanical oscillations, and failure in ripple control. The main sources of interharmonic waveform distortion are static frequency converters, cycle-converters, induction motors, and arcing devices. Furthermore, in order to verify the performance of the proposed method, 100 signals with various characteristics of harmonics and inter-harmonics accordingly with refer to IEEE Std. 1159-2009 are used respectively.

\section{LINEAR TIME-FREQUENCY DISTRIBUTIONS}

Time-frequency distributions are great techniques that represent a signal in, jointly, time and frequency representation as known as time-frequency representation (TFR). From TFR, spectral information of a signal can be observed with changes of time [8]. Therefore, the TFD are very applicable to analyse power quality signals that consist of non-stationary and multi-frequency components signal. In the following sections, one of TFDs which is discussed.

By windowing the signal at first and after that taking the Fourier transforms the time-localization can be obtained correctly. Furthermore, this will lead to the rise of short time Fourier transform, (STFT) or windowed Fourier transform [9]. The continuous-time STFT of a signal, $x(t)$, is expressed as

$$
\rho_{x}(t, f)=\int_{-\infty}^{\infty} x(\tau) w(\tau-t) e^{-j 2 \pi f \tau} d \tau
$$

where $x(\tau)$ is the signal which is used as an input and $w(\tau)$ is the window which is used as an observation. However, the squared value of the STFT is, usually used in the signal analysis which called as a spectrogram. The analysis of spectrogram is represented in TFR that represents three-dimensional imitation of the signal energy when compared with time and frequency and is shown mathematically as: 


$$
P_{x}(t, f)=\left|\int_{-\infty}^{\infty} x(\tau) w(\tau-t) e^{-j 2 \pi f \tau} d \tau\right|^{2}
$$

In this approach, the Hanning window is chosen due to its lower peak side lope which is the narrow effect on other frequencies around fundamental value which is $50 \mathrm{~Hz}$ and other frequency components. The Hanning window function can be formulated as:

$$
w(t)=\frac{1}{2}\left(1-\cos \frac{2 \pi t}{T}\right)
$$

\section{SIGNAL PARAMETERS}

Parameters of signals of voltage variation are estimated from the TFR. The signal constituents are momentary RMS voltage and RMS fundamental voltage, instantaneous total waveform distortion (TWD), instantaneous total harmonic distortion (THD) and instantaneous total inter-harmonic distortion (TnHD) respectively. The following are parameters of signal that been obtained from TFR [10].

Instantaneous RMS Voltage, $V_{r m s}(t)=\sqrt{\int_{0}^{f_{s}} P_{x}(t, f) d f}$

Instantaneous RMS Fundamental Voltage, $V_{1_{r m s}}(t)=\sqrt{2 \int_{f_{l o}}^{f_{h i}} P_{x}(t, f) d f}$

Instantaneous Total Waveform Distortion, $T W D(t)=\frac{\sqrt{V_{r m s}(t)^{2}-V_{1 r m s}(t)^{2}}}{V_{1_{r m s}}(t)}$

Instantaneous Total Harmonic Distortion, $\operatorname{THD}(t)=\frac{\sqrt{\sum_{h=2}^{H} V_{h, r m s}(t)^{2}}}{V_{1 r m s}(t)}$

Instantaneous Total Nonharmonic Distortion, $\operatorname{Tn} H D(t)=\frac{\sqrt{V_{r m s}(t)^{2}-\sum_{h=0}^{H} V_{h, r m s}(t)^{2}}}{V_{1 r m s}(t)}$

where $\operatorname{Px}(t, f)$ is the TFR of a signal, $f_{s}$ is sampling frequency, $f_{0}$ is the fundamental frequency, $V_{l r m s(t)}$ is instantaneous RMS fundamental voltage, $V_{r m s(t)}$ is instantaneous RMS voltage and $V_{h, r m s}(t)$ is RMS harmonic voltage. $f_{h i}=f_{0}+25 \mathrm{~Hz}, f_{l o}=f_{0}-25 \mathrm{~Hz}, 25 \mathrm{~Hz}$ is chosen for $f_{h i}$ and $f_{l o}$, it can represent the fundamental frequency value and use for calculate the value of the frequency element.

\section{SIGNAL CHARACTERISTIC}

The determination of signal characteristics is from the calculated signal parameters. Furthermore, by using the instantaneous RMS voltage, the signal properties for example the average of RMS voltage can be calculated using equation 9 [11]. The information of the signal characteristics is used as input for classifier to allocate the harmonic and inter-harmonic signals.

$$
V_{r m s, a v e}=\frac{1}{T} \int_{0}^{T} V_{r m s}(t) d t
$$


Average of total harmonic distortion, $\mathrm{THD}_{\mathrm{ave}}$ and total nonharmonic distortion, $\mathrm{TnHD}_{\mathrm{ave}}$ can be calculated from instantaneous total harmonic distortion, $\mathrm{THD}(\mathrm{t})$ and instantaneous total nonharmonic distortion, $\operatorname{TnHD}(\mathrm{t})$, respectively. They can be defined as:

$$
\begin{aligned}
& T H D_{\text {ave }}=\frac{1}{T} \int_{0}^{T} T H D(t) d t \\
& T n H D_{\text {ave }}=\frac{1}{T} \int_{0}^{T} \operatorname{Tn} H D(t) d t
\end{aligned}
$$

\section{SIGNAL CLASSIFICATION}

The rule-based classifier is a deterministic classification method that has been, widely, used in real world application. The classification performance is much reliant on the best rules and threshold values [6]. In this analysis, since the signal characteristics give good previous information for the power quality signals, the rule-based classifier which is rule is appropriate to be utilized for signal classification. The rule-based classifier that been used in order to identify harmonics and inter-harmonics as in equation 12 and 13 [12].

$$
\begin{aligned}
& T H D_{\text {ave }}>T n H D_{\text {thres }} \text { and } T n H D_{\text {ave }}<T n H D_{\text {thres }} \\
& T n H D_{\text {ave }}>=T n H D_{\text {thres }} \text { and } T H D_{\text {ave }}<T n H D_{\text {thres }}
\end{aligned}
$$

Meanwhile, the flow chart in Figure 1 shows a process of rule-based classifier of the harmonics and inter-harmonics signals. Harmonic and inter-harmonic, their threshold value, $\mathrm{THD}_{\text {thres }}$ and $\mathrm{TnHD}_{\text {thres }}$ are set based on several analyses made for these signals. THD or TnHD considered exist in the signal as their magnitude is greater or equal than the threshold value. Thus, harmonic signal is classified as a signal that has only THD while inter-harmonic signal has only TnHD.

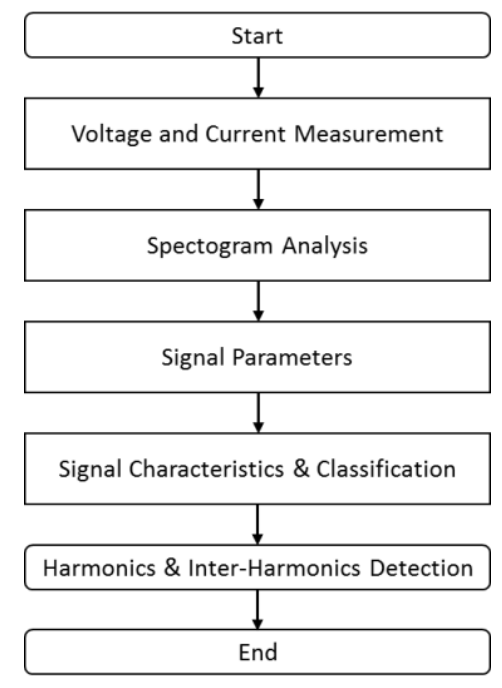

Figure 1. The Implementation Flow Chart for Harmonics and Inter-Harmonics Detection

\section{ACCURACY MEASUREMENT}

The performance and viability of proposed method depend on the accuracy of this technique and the evaluation of accuracy can be done as follows.

The accuracy of the analysis is identified by measuring the signal characteristics measurement accuracy. The characteristics measurement is very important because it will be used as input for the classifier of the signal. Low accuracy of the measurement will result in misinterpretation in exact signal characteristics. To measure the exactness of the measurement, mean absolute percentage error (MAPE) is used as an index [13]. 
It can be defined as:

$$
M A P E=\frac{1}{N} \sum_{n=1}^{N}\left|\frac{x_{i}(n)-x_{m}(n)}{x_{i}(n)}\right| x 100 \%
$$

where $x_{i}(n)$ is an actual value, $x_{m}(n)$ is measured value and $\mathrm{N}$ is the number of data. The smaller value of MAPE offers more accurate results.

\section{RESULTS AND ANALYSIS} spectrogram.

This section will discuss the results for harmonics and inter-harmonics detection technique utilizing

\subsection{Detection of Harmonic Signal}

Figure 2(a) and (b) present harmonic signal in time domain and its TFRs using spectrogram. The TFRs indicate that the signal consists of two frequency component: fundamental frequency $(50 \mathrm{~Hz})$ and $7 \mathrm{th}$ harmonic component $(350 \mathrm{~Hz})$. Signal parameters estimated from the TFR using spectrogram is shown in Figure 2(b). Figure 2(c) shows that the harmonic voltage contributes to the increases of RMS voltage from normal voltage which is 1.0 to $1.17 \mathrm{pu}$. However, it does not change the RMS fundamental voltage which remains constant at $1.0 \mathrm{pu}$. Besides that, the signal also results the magnitude of TWD and THD remains constant at $60 \%$ and zero percent for the TnHD as shown in Figure 2(d). Thus, the detection of harmonic signal successfully carried out using the spectogram technique.

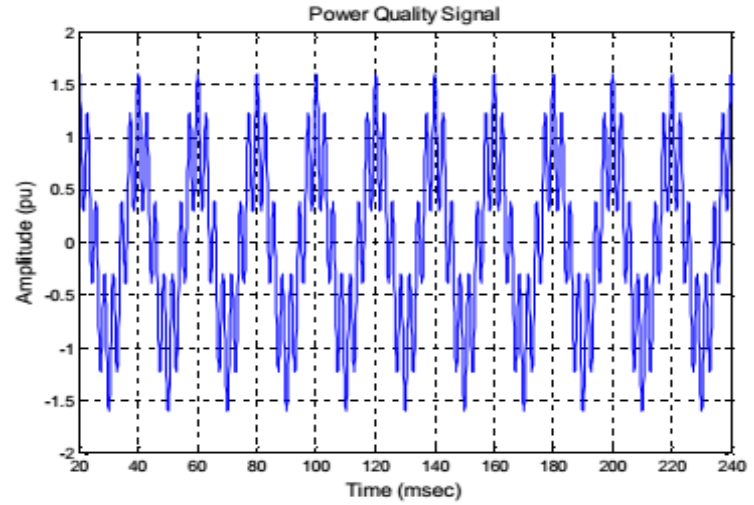

(a)
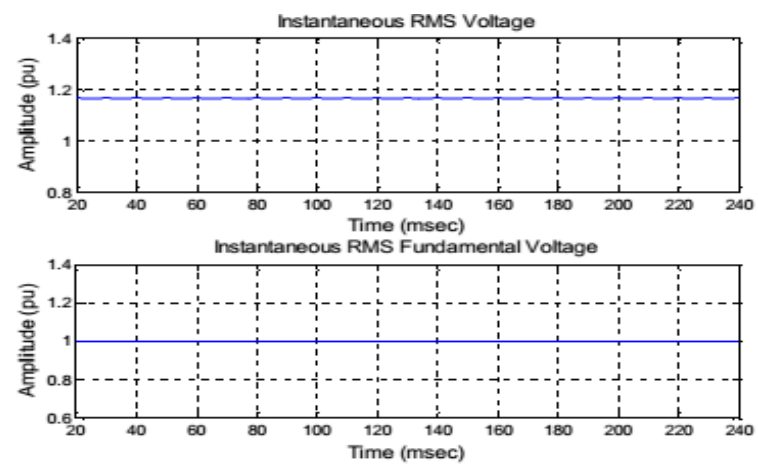

(c)

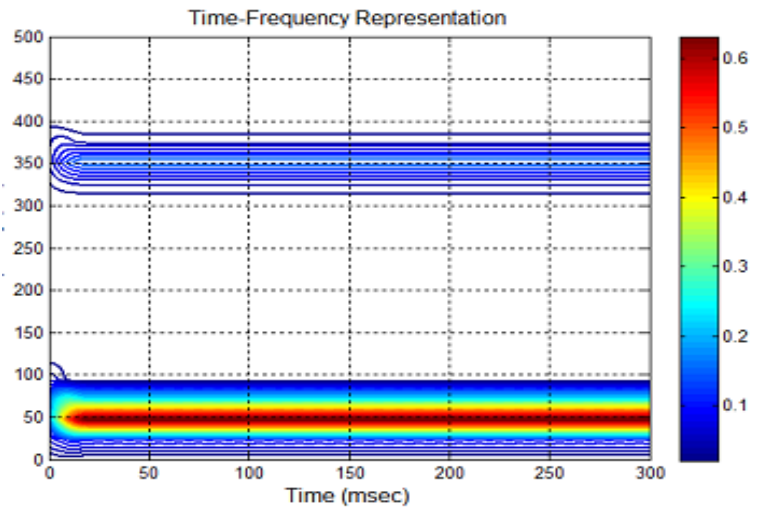

(b)
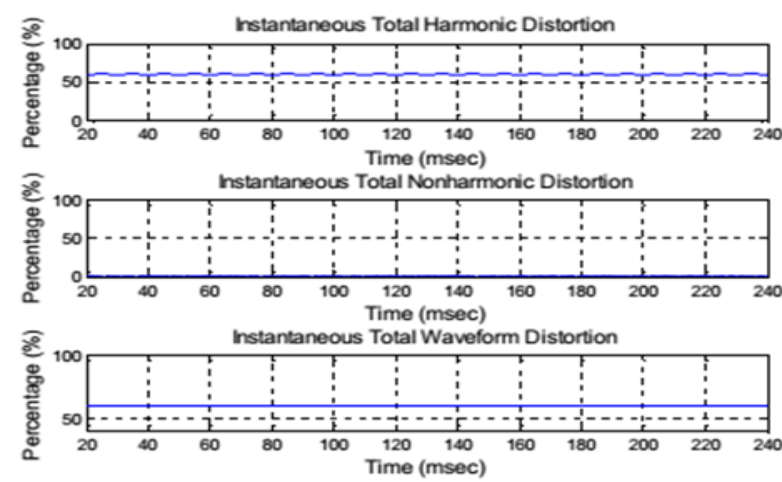

(d)

Figure 2. (a) Harmonic Signal from Simulation and its, (b) TFR using Spectrogram, (c) Instantaneous of RMS and Fundamental RMS Voltage, (d) Instantaneous of Total Harmonic Distortion, Total Nonharmonic Distortion And Total Waveform Distortion 


\subsection{Detection of Inter-Harmonic Signals}

Inter-harmonic signal and its TFR using spectrogram is shown in Figure 3(a) and (b), respectively. As shown in Figure 3(b), the signal has two frequency components which are at fundamental frequency $(50 \mathrm{~Hz})$ and inter-harmonic frequency of $375 \mathrm{~Hz}$. Signal parameters estimated from the TFR using spectrogram are shown in Figure 3(b). Figure 3(c) shows that the inter-harmonic voltage give rise to the increases of RMS voltage from normal voltage from 1.0 to $1.17 \mathrm{pu}$. However, it does not change the RMS fundamental voltage which remains constant at $1.0 \mathrm{pu}$. Besides that, the signal also results the magnitude of TWD and TnHD remains constant at $60 \%$ and zero percent for the THD as shown in Figure 3(d). The detection of inter-harmonic signal magnificently accomplished using the proposed technique.

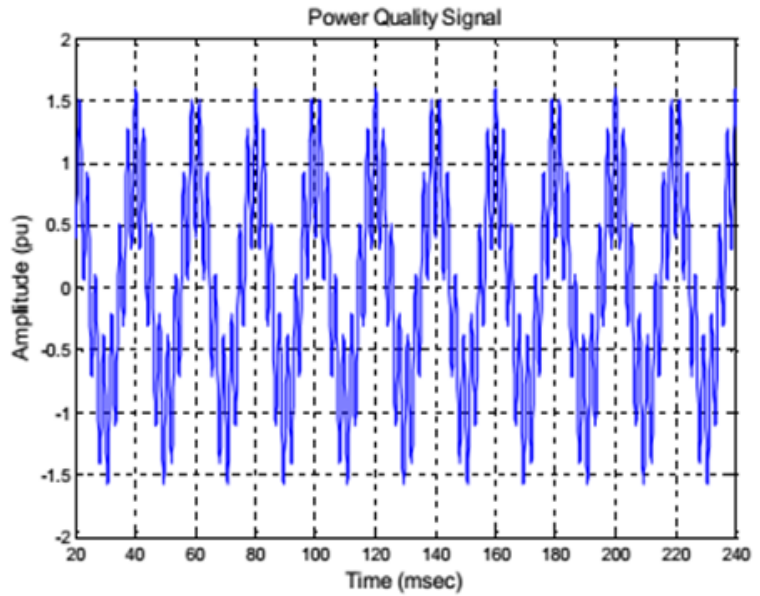

(a)
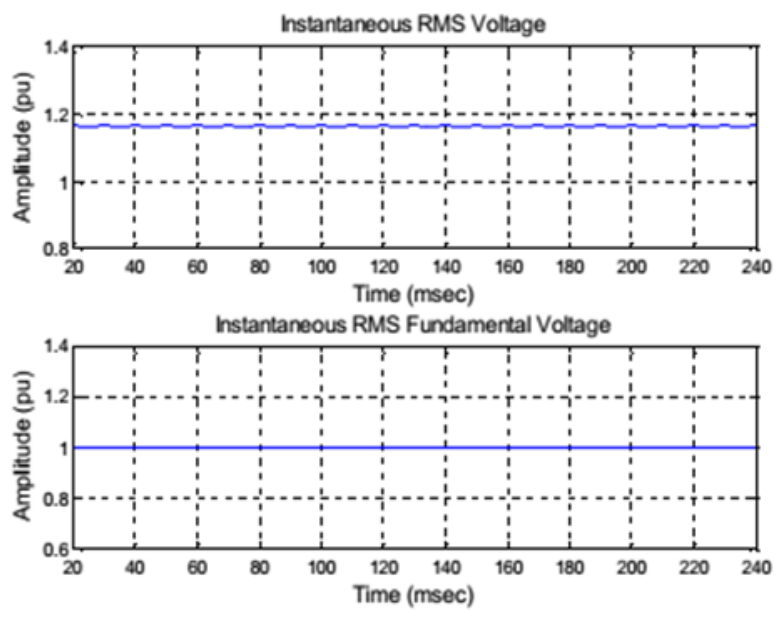

(c)

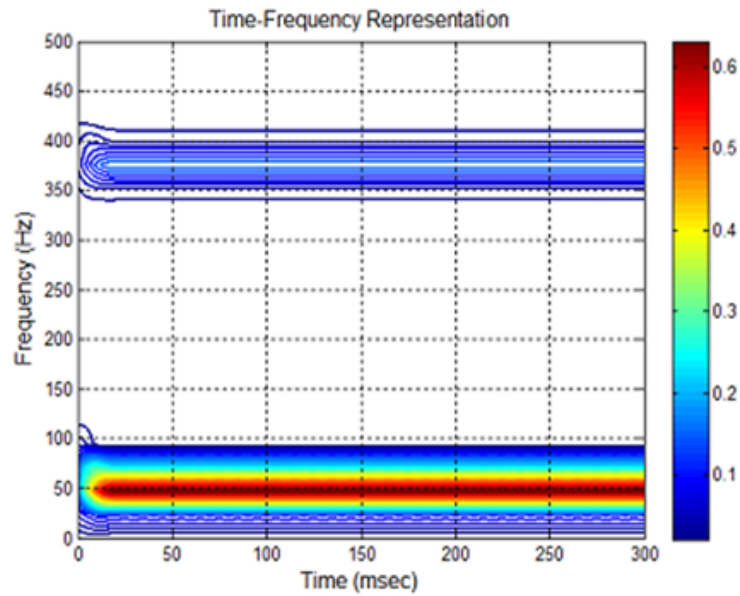

(b)
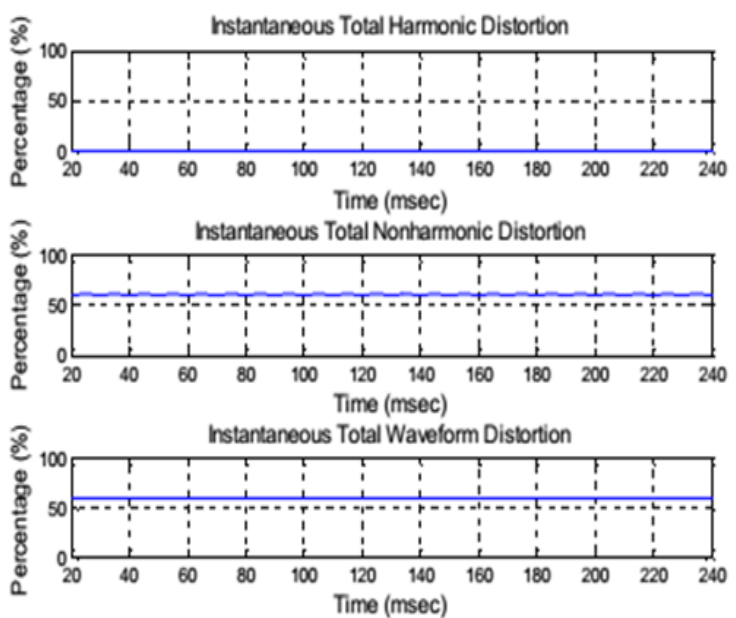

(d)

Figure 3. (a) Interharmonic Signal from Simulation and its, (b) TFR using Gabor Transform, (c) Instantaneous of RMS and Fundamental RMS Voltage, (d) Instantaneous of Total Harmonic Distortion, Total Nonharmonic Distortion and Total Waveform Distortion

\subsection{The Accuracy of the Analysis}

Harmonic and inter-harmonics signals are tested and mean absolute percentage error (MAPE) of the signal properties are calculated. Then, the results are averaged to identify the accuracy of the measurements as shown in Table 1. The table shows that spectogram gives good accuracy for an average of RMS voltage, THD and TnHD. In addition, spectogram has a good accuracy in harmonic and inter-harmonic signals detection. 


\begin{tabular}{cc} 
Table 1. MAPE Simulation Result for Spectrogram Analysis \\
\hline Signal Characteristics & MAPE $(\%)$ \\
\hline $\mathrm{V}_{\text {rms.ave }}$ & 0.1572 \\
THD $_{\text {ave }}$ & 0.1551 \\
TnHD ave $^{\text {and }}$ & 0.1595 \\
\hline
\end{tabular}

\subsection{Detection and Classification of Harmonic and Inter-Harmonic Signals}

As shown in the previous section, spectogram has high accuracy in identifying the signal characteristics. Furthermore, the performance results of the signals classification using the spectogram are shown in Table 2. 100 signals with various characteristics for each type of voltage signal is generated and classified. The table shows that the classification results using spectogram give $100 \%$ correct classification for all signals.

Table 2. Performance of Harmonic and Inter-Harmonic Signals Classification

\begin{tabular}{ccc}
\hline Signal & \multicolumn{3}{c}{ Gabor Transform } \\
& Number of data sets & 100 \\
Harmonics & 100 & 100 \\
Inter-harmonics & 100 & 100 \\
Normal & 100 & Correct Classification \\
\hline
\end{tabular}

\section{CONCLUSION}

The performance evaluation of the signal analysis using spectogram are implemented in time domain as well as frequency domain in terms of accuracy using MAPE. Furthermore, the verification of detection and classification of harmonic signals are utilizing 100 signals with various characteristics for each type of voltage signals. The results show that the spectogram gives good accuracy and gives 100 percent correctness of signals classification. Hence, spectogram is an appropriate technique to be implemented for harmonic signals detection and classification in power distribution system.

\section{ACKNOWLEDGEMENTS}

This research is supported by Advance Digital Signal Processing Laboratory (ADSP Lab). Special thanks also to the Faculty of Electrical Engineering and Engineering Technology of Universiti Teknikal Malaysia Melaka (UTeM), Ministry of Higher Education Malaysia (MOHE) and Ministry of Science, Technology and Innovation (MOSTI) for giving the cooperation and funding for this research with grant number 06-01-14-SF00119 L00025. Their support is gratefully acknowledged.

\section{REFERENCES}

[1] S. Khokhar, et al., "Automatic Classification of Power Quality Disturbances : A Review," IEEE Student Coference Res. Dev., no. December, pp. 16-17, 2013.

[2] O. Ozgonenel, et al., "A new classification for power quality events in distribution systems," Electr. Power Syst. Res., vol. 95, pp. 192-199, 2013.

[3] M. Gupta, et al., "Neural Network Based Indexing and Recognition of Power Quality Disturbances," TELKOMNIKA (Telecommunication Comput. Electron. Control., vol/issue: 9(2), pp. 227-236, 2011.

[4] S. A. Deokar and L. M. Waghmare, "Integrated DWT-FFT approach for detection and classification of power quality disturbances,” Int. J. Electr. Power Energy Syst., vol. 61, pp. 594-605, 2014.

[5] M. V. Rodriguez, et al., "Detection and classification of single and combined power quality disturbances using neural networks," IEEE Trans. Ind. Electron., vol/issue: 61(5), pp. 2473-2482, 2014.

[6] A. A. Ahmad and A. Zuri, "Analysis and Classification of Airborne Radar Signal Types Using Time-Frequency Analysis," vol/issue: 2014(September), pp. 23-25, 2014.

[7] N. H. H. Abidullah, et al., "Real-Time Power Quality Disturbances Detection and Classification System," World Appl. Sci. J., vol/issue: 32(8), pp. 1637-1651, 2014.

[8] M. Manap, et al., "Comparison of Open and Short-Circuit Switches Faults Voltage Source Inverter (VSI) Analysis Using Time-Frequency Distributions," Appl. Mech. Mater., vol/issue: 752-753(APRIL), pp. 1164-1169, 2015.

[9] A. R. Abdullah, et al., "Short-circuit switches fault analysis of voltage source inverter using spectrogram," Electr. Mach. Syst. (ICEMS), 2013 Int. Conf., pp. 1808-1813, 2013.

[10] A. R. Abdullah, et al., "Power Quality Monitoring System Utilizing Periodogram And Spectrogram Analysis Techniques," Int. Conf. Control. Instrum. Mechatronics Eng., no. August, pp. 770-774, 2007.

[11] N. A. Abidullah, et al., "Real-time power quality signals monitoring system," Proceeding - 2013 IEEE Student 
Conf. Res. Dev. SCOReD 2013, no. December, pp. 433-438, 2015.

[12] A. R. Abdullah, et al., "Performance Evaluation of Real Power Quality Disturbances Analysis Using S-Transform," Appl. Mech. Mater., vol. 752-753, pp. 1343-1348, 2015.

[13] K. R. Cheepati and T. N. Prasad, "Performance Comparison of Short Term Load Forecasting Techniques," Int. J. Grid Distrib. Comput., vol/issue: 9(4), pp. 287-302, 2016.

\section{BIOGRAPHIES OF AUTHORS}
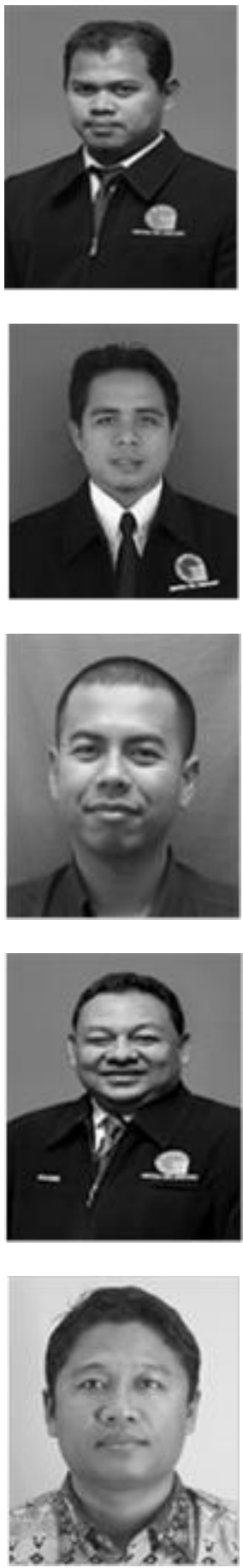

Mohd Hatta Jopri was born in Johor, Malaysia on 1978. He received his B.Sc from Universiti Teknologi Malaysia in 2000 and Msc. in Electrical Power Engineering from RheinischWestfälische Technische Hochschule Aachen (RWTH), Germany in 2011. Since 2005, he has been an academia staff in the Universiti Teknikal Malaysia Melaka (UTeM) and currently he pursuing his $\mathrm{PhD}$ in the area of power quality.

Dr. Abdul Rahim Abdullah was born in Kedah, Malaysia on 1979. He received his B. Eng., Master Eng., PhD Degree from Universiti Teknologi Malaysia in 2001, 2004 and 2011 in Electrical Engineering and Digital Signal Processing respectively. He is currently an Associate Professor with the Department of Electrical Engineering, Chief of Advanced Digital Signal Processing (ADSP) Lab and Center of Excellent (COE) Coordinator for Universiti Teknikal Malaysia Melaka (UTeM).

Mustafa Manap was born in Kuala Lumpur, Malaysia on 1978. He received his B.Sc from Universiti Technologi Malaysia in 2000 and Msc. in Electrical Engineering from Universiti Teknikal Malaysia Melaka (UTeM) 2016. Since 2006, he has been an academia staff in the Universiti Teknikal Malaysia Melaka (UTeM). His research interests are power electronics and drive, instrumentation, and DSP application.

Mohd Rahimi bin Yusoff was born in Kelantan, Malaysia on 1967. He received his M.Sc. in Electrical Engineering from University of Nottingham, England in 1992 and B.Eng. in Electrical \& Electronics from University of Wales, Swansea, UK in 1990. Since 2010, he has been an academia staff in Universiti Teknikal Malaysia Melaka (UTeM) and currently holding the post as Dean of Faculty of Engineering Technology. His research interests are power quality, signal processing, and DSP application.

Tole Sutikno is an Associated Professor in Department of Electrical Engineering at Universitas Ahmad Dahlan (UAD), Indonesia. He is Editor-in-Chief, Principle Contact and Editor for some Scopus indexed journals. He is also one of founder of the Indonesian Publication Index (IPI). He has completed his studies for B.Eng., M.Eng., Ph.D. in Electrical Engineering from Diponegoro University, Gadjah Mada University and Universiti Teknologi Malaysia, respectively. His research interests include the field of industrial electronics and informatics, power electronics, FPGA applications, embedded systems, data mining, information technology and digital library. 


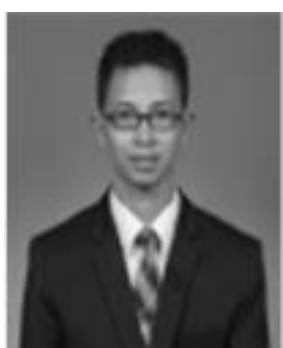

Mohd Faiz bin Habban was born in Perak, Malaysia on 1987. He graduated with Bachelor of Electrical Engineering Technology in Power Industry (Hons.) from Universiti Teknikal Malaysia Melaka. He has doing the industrial training at Malakoff Berhad, Prai Power Sdn Bhd and working at Masterplan Consulting Sdn Bhd, as Junior Engineer. Currently, he pursuing his Master at Universiti Teknikal Malaysia Melaka (UTeM). His area of interest is Electrical Power Engineering, Power Electronics \& Drive, Digital Signal Processing and Energy Efficiency. 